\title{
Retomar la agenda del desarrollo
}

\author{
José Antonio Ocampo \\ Secretario Ejecutivo de la \\ Comisión Económica para \\ América Latina y el Caribe \\ (CEPAL) \\ Jaocampo@eclac.cl
}

En este ensayo se resumen algunas de las reflexiones más importantes en que se sustentan las propuestas a favor de una nueva agenda de desarrollo y se resalta la necesidad de lograr un nuevo equilibrio entre el mercado y el interés público y de entender por "políticas públicas" toda forma de acción organizada tras objetivos de interés común, y no sólo las acciones estatales. El autor plantea cinco propuestas a favor de una nueva agenda de desarrollo: i) Se requiere una globalización más equilibrada, con verdadero respeto por la diversidad; la existencia de una red de instituciones regionales, respetuosas del orden global pero con mayores grados de autonomía, constituye la mejor opción para construir una institucionalidad internacional más sólida y equilibrada. ii) Es fundamental tener una visión amplia de la estabilidad macroeconómica y del papel que desempeñan las políticas anticíclicas; está claro que la inestabilidad real tiene un costo muy elevado y que, además, el costo de los déficit del sector privado es tan alto como el de los desequilibrios del sector público. iii) No basta con las políticas macroeconómicas; la definición de estrategias de desarrollo que apunten a inducir innovaciones y construir complementariedades productivas parece ser un camino que las economías abiertas de la región no pueden dejar de tomar. iv) Es preciso mejorar los encadenamientos sociales; el progreso social puede concebirse como el producto de tres factores básicos: una política social de largo plazo que incremente la equidad y garantice la inclusión, un crecimiento económico que genere un volumen adecuado de empleo de calidad y una reducción de la heterogeneidad estructural de los sectores productivos. v) El sistema económico debe estar subordinado a objetivos sociales más amplios; en este sentido, es importante fomentar los lazos de solidaridad que se han ido perdiendo. 


\section{I}

\section{Introducción}

La liberalización económica fue presentada al mundo en desarrollo como la respuesta a estrategias ineficientes asociadas a la protección comercial, a los altos niveles de intervención estatal y a la captación de rentas (rent seeking) por parte de los agentes económicos, que dichas estrategias generaban. Fue presentada también como la forma de aprovechar plenamente las oportunidades que ofrecía la globalización. Esta visión representó un cambio significativo frente a la idea que sirvió de base a las estrategias de desarrollo durante varias décadas, según la cual la "industrialización tardía" exigía una amplia intervención del Estado. El Consenso de Washington fue uno de los mejores resúmenes de esta agenda de reformas, aunque por cierto no representaba las versiones más radicales, que reclamaban un Estado minimalista (Williamson, 1998). Igualmente, fue una manifestación del optimismo que despertaba la agenda de reformas hace una década.

En los últimos años se ha producido un intenso cuestionamiento acerca de la sabiduría de esta visión. Es probable que la crisis asiática haya sido su peor revés, al hacer patente que la liberalización financiera puede ser fuente de una profunda inestabilidad macroeconómica cuando no está acompañada de un marco institucional apropiado. La fuerte reacción de la "sociedad civil global" a partir de Seattle revela que la globalización misma está siendo cuestionada, lo que refleja básicamente el descontento existente en el mundo industrializado. En los países en desarrollo, la desilusión con las reformas es creciente, pero sus manifestaciones políticas son menos organizadas y su agenda está todavía por definir.

En términos más generales, la insatisfacción con los resultados de las reformas es cada vez mayor. El comercio y la inversión extranjera directa han crecido notablemente, pero la "Tierra prometida" de altas tasas de crecimiento se percibe cada vez más como un espejismo. En América Latina, la región en donde más se ha avanzado en el proceso de reformas, el crecimiento de los años noventa fue tan sólo de un $3.2 \%$ anual,

$\square$ Una versión previa de este ensayo se presentó en el panel de la reunión anual de la American Economic Association "Toward a Post-Washington Consensus on Development and Security", realizada en Nueva Orleans del 5 al 7 de enero de 2001. ritmo significativamente inferior a las cifras registradas durante las tres décadas de industrialización liderada por el Estado, entre los años cincuenta y setenta (5.5\% por año). El desempeño del Africa subsahariana y, en general, de los países menos desarrollados, sigue siendo muy insuficiente. Varias de las economías en transición mantienen niveles de actividad económica inferiores a los registrados antes de la "gran eclosión". La mayor parte de las economías asiáticas afectadas por la crisis ya se han recuperado, pero aún tienen que enfrentar las repercusiones de las crisis financieras. Las excepciones más notables son, como es obvio, China e India, que no figuran en todo caso entre las economías con mayores niveles de reforma. Incluso en el mundo industrializado el crecimiento de los años noventa fue muy inferior al de la "edad de oro" (1950 a 1973); los Estados Unidos alcanzaron las tasas de crecimiento que caracterizaron dicho período, pero sólo en la segunda mitad de la década.

Por otra parte, las tensiones distributivas son agudas y es probable que estén aumentando. Las disparidades de ingreso entre los países desarrollados y los menos desarrollados siguen acentuándose (PNUD, 1999). La distribución del ingreso se ha deteriorado en un gran número de países, tanto desarrollados como en desarrollo. De acuerdo con un cálculo existente, el $57 \%$ de la población mundial vive en países cuya distribución del ingreso se ha deteriorado, frente a sólo un $16 \%$ en aquellos en que ha mejorado, mientras en los demás no se observa ninguna tendencia definida (Cornia, 1999). El aumento de los diferenciales de ingreso en función del grado de calificación de la mano de obra es un fenómeno de alcance mundial, aunque sus explicaciones alternativas - la liberación del comercio, tendencias tecnológicas o el debilitamiento de las instituciones de protección social- siguen siendo objeto de debate. No obstante, es indudable que obedece, al menos en parte, a la asimetría que existe entre la movilidad internacional de algunos factores de producción (el capital, la mano de obra altamente calificada) y las restricciones a la movilidad de otros (mano de obra poco calificada), así como a la creciente dificultad de los gobiernos para proporcionar instrumentos eficaces de protección social (Rodrik, 1997).

Estos hechos y el descontento que provocan han dado origen, a su vez, a un debate positivo, que segura- 
mente enriquecerá la agenda del desarrollo. En efecto, en los últimos años este debate ha adquirido un carácter algo más pluralista, del cual empiezan a surgir nuevas visiones del desarrollo. Nuevos énfasis -entre otros en la importancia del desarrollo institucional, en las redes de protección social, en el "sentido de pertenencia" (ownership) de las políticas de desarrollo - han penetrado con fuerza en el debate internacional. ¿Significa esto que la agenda del desarrollo está cambiando? Es posible, pero aún no es evidente. En muchos casos, los nuevos conceptos y énfasis son simplemente añadidos a lo que sigue siendo esencialmente la misma agenda de los últimos años, en una concepción lineal según la cual se van agregando nuevas "generaciones" de reformas a las que se consideran buenos cimientos. En las condiciones menos favorables, son exclusivamente nuevos ropajes para las mismas ideas. Los mercados, en particular los financieros, no han internalizado realmente la necesidad de una nueva agenda de desarrollo y en esencia siguen reclamando una mayor liberalización, tanto a nivel nacional como mundial, es decir simple y llanamente más reformas de "primera generación". Esta sigue siendo la fuerza dominante en un mundo en el cual la capacidad de acción de los gobiernos nacionales se ha debilitado y hay tan sólo una transición precaria hacia políticas globales.

En el presente ensayo se resumen algunas de las reflexiones más importantes en las que se fundamentan las propuestas a favor de una nueva agenda de desarrollo. ${ }^{1}$ Dos temas transversales que permean la literatura existente deben resaltarse desde un comien- zo. El primero es el llamado a un nuevo equilibrio entre el mercado y el interés público. Este llamado no debe ser visto como contrario al mercado, ya que las acciones en favor de la provisión de bienes públicos, de completar mercados, de ayudar a que los mercados no competitivos funcionen adecuadamente, de explotar las externalidades positivas y evitar las negativas, o de garantizar una participación equitativa en los frutos del desarrollo, pueden operar, a través de diferentes canales económicos, sociales y políticos, como poderosos mecanismos en beneficio del mercado. Si es aplicada correctamente, una política activa de este tipo será más "amistosa con el mercado" que los enfoques alternativos que tendieron a predominar durante la primera ola de reformas.

El segundo tema es que las "políticas públicas" deben ser entendidas como toda forma de acción organizada en favor de objetivos de interés común, más que exclusivamente como acciones estatales. Este concepto es consistente con el llamado a abrir nuevas oportunidades para la participación de la sociedad civil y con la necesidad de superar la crisis del Estado que afecta al mundo en desarrollo y, en realidad, al mundo entero. Busca corregir tanto las "fallas del mercado" como las "fallas del gobierno" y, más en general, busca construir y reconstruir instituciones (o, en la terminología de la nueva literatura institucional, instituciones y organizaciones), sin duda una de las tareas más complejas que enfrentan las economías en desarrollo y en transición hoy e, incluso, la tarea más urgente y aún insuficientemente abordada en el proceso de construir un mejor orden internacional.

\section{II}

\section{Una globalización más equilibrada con verdadero respeto por la diversidad}

La necesidad de "civilizar" la economía global (Helleiner, 2000) o, como se expresó en la Declaración del Milenio de las Naciones Unidas, de "conseguir que la mundialización se convierta en una fuerza positiva para todos los habitantes del mundo" (Naciones Unidas, 2000) es, sin duda, el tema más importante. Aunque

1 Aunque la literatura sobre el tema es extensa, las referencias se han limitado a un mínimo. la globalización responde a procesos tecnológicos y económicos dinámicos, no cabe duda de que puede ser moldeada. De hecho, la forma que ha ido adoptando ha estado determinada, en gran medida, por decisiones explícitas de política.

En este sentido, el rasgo más preocupante es el carácter incompleto, e incluso desequilibrado, del actual proceso de globalización y de la agenda internacional de políticas que lo acompaña, que reproduce 
antiguas asimetrías de la economía mundial y crea otras nuevas. Hay cuatro aspectos que predominan en la actual agenda: el libre comercio, los derechos de propiedad intelectual, la protección de las inversiones, y la liberalización financiera y de la cuenta de capital. A esta última se le han impuesto ciertas condiciones adicionales a raíz de las crisis más recientes: debe realizarse siguiendo una secuencia adecuada, y se debe otorgar prioridad a los flujos a largo plazo y al desarrollo institucional. Más aun, en el ámbito del comercio, la liberalización es incompleta y asimétrica: los productos "sensibles" de gran interés para los países en desarrollo están sujetos a los mayores niveles de protección en el mundo industrializado.

Por el contrario, otros aspectos han sido claramente marginados de la agenda actual: la movilidad de la mano de obra; las normas internacionales sobre tributación, especialmente sobre el capital, esenciales para garantizar la tributación adecuada de este factor de alta movilidad; la formulación de normas auténticamente internacionales sobre competencia y códigos de conducta aplicables a las empresas multinacionales, y el financiamiento compensatorio para asegurar la incorporación de aquellos países y grupos sociales que tienden a quedar rezagados en el proceso de globalización.

Lo anterior refleja, a su vez, la asimetría más importante: la que existe entre la rápida globalización de (algunos) mercados y la notoria ausencia de una verdadera agenda social internacional. Esta última se limita a la definición de principios internacionales comunes (en cumbres de las Naciones Unidas) y a una incipiente legislación internacional. La reducción de la asistencia oficial para el desarrollo es la demostración más evidente de la falta de un adecuado compromiso con una agenda social auténticamente internacional, al igual que la creciente condicionalidad que caracteriza el apoyo financiero internacional en general.

En términos más amplios, se reconoce crecientemente que la globalización ha hecho más evidente la necesidad de proporcionar ciertos "bienes públicos globales" (políticos, sociales, económicos y ambientales), en vista de que muchos bienes públicos que antes eran "nacionales" (y, mucho antes, "locales") han ido transformándose en bienes públicos globales (Kaul, Grunberg y Stern, comps., 1999). Sin embargo, hay un indiscutible contraste entre el reconocimiento de este hecho y la debilidad de las estructuras internacionales destinadas a proporcionar este tipo de bienes y los fondos que se les asignan para cumplir esa función.

Estas asimetrías reflejan los rasgos fundamentales de la política y la economía política del mundo de hoy. No cabe duda de que el desequilibrio del proceso y de la agenda actual de globalización es un reflejo de la mayor influencia que ejercen los países más grandes y las grandes empresas multinacionales. Pero también refleja la desorganización de los actores, particularmente de los países en desarrollo, en los debates internacionales. Este comportamiento no sólo está vinculado al debilitamiento de los mecanismos históricos de acción concertada entre los países en desarrollo (entre otros, el Grupo de los 77), sino también a la "competencia de políticas" que ha generado la globalización misma: el incentivo a que cada uno de los países se muestre individualmente como el más atractivo para las inversiones, en una época de movilidad del capital y de creciente producción susceptible de relocalización. Por lo tanto, las asimetrías en las relaciones mundiales de poder y el alto costo de establecer coaliciones internacionales para compensarla han adquirido cada vez mayor importancia.

La situación se ve indudablemente afectada por un elemento de política y economía política: la resistencia de la mayoría de los países a ceder su soberanía económica a las organizaciones internacionales. Bajo las vigorosas fuerzas del mercado que caracterizan a la globalización y tienden a debilitar a los Estados-nación, y los procesos unilaterales de liberalización emprendidos simultáneamente por los países, las regulaciones gubernamentales se han debilitado en el mundo entero. Para muchos analistas esto constituye un adelanto, pero también es una fuente de graves distorsiones y riesgos, sobre todo, aunque no exclusivamente, en el ámbito financiero. Además, si bien el regionalismo abierto es uno de los rasgos característicos de la globalización y en muchas regiones del mundo en desarrollo (entre otras América Latina y el sudeste de Asia) se han desplegado grandes esfuerzos de integración, éstos no se han traducido en fuertes coaliciones entre países en desarrollo. De hecho, fuera de la Unión Europea, los países no están dispuestos a ceder su soberanía ni a organizaciones regionales (y en la Unión Europea sólo en forma limitada).

Estas características de la política y de la economía política tienen importantes consecuencias para la reforma internacional. La más evidente es que los esfuerzos por lograr una reforma sustantiva serán débiles, lo que posiblemente impedirá responder a la necesidad de una globalización equilibrada y una mejor gobernabilidad mundial. Además, hace más difícil un proceso equilibrado de negociación y podría conducir a que se subestimen o ignoren por completo los intere- 
ses de algunos actores. La falta de instituciones auténticamente internacionales también implica que las instituciones que se han creado en el pasado a nivel nacional no existirán a nivel global o sólo tendrán funciones muy limitadas.

Los arreglos internacionales incompletos que se pueden prever indican que los actores débiles deben seguir reclamando autonomía nacional en áreas de importancia crítica, en particular la definición de la estrategia de desarrollo económico y social. Más aun, la autonomía nacional es el único sistema coherente con el fomento de la democracia a nivel mundial. En efecto, no tiene ningún sentido fomentar la democracia si no se permite que los procesos nacionales de representación y participación influyan en la determinación de las estrategias de desarrollo económico y social. Lo anterior concuerda con la idea de que el desarrollo institucional, la cohesión social y la acumulación de capital humano y capacidad tecnológica ("capital conocimiento") son procesos esencialmente endógenos. Empleando un término del estructuralismo latinoamericano, el desarrollo sólo puede darse "desde dentro" (Sunkel, 1991). El apoyo a estos procesos endógenos, el respeto de la diversidad y la formulación de normas que le permitan florecer son elementos esenciales de un orden mundial democrático orientado al desarrollo.

Una última y crucial consecuencia de lo anterior es que ninguna arquitectura internacional es neutra en términos de equilibrio en las relaciones internacionales. En este sentido, un sistema internacional que dependa de unas pocas instituciones de alcance mundial será menos equilibrado que un sistema basado en una red de instituciones regionales y la posición de los países con muy escaso poder en el ámbito internacional mejoraría si participaran activamente en los esquemas regionales. De hecho, estos esquemas pueden ofrecer un grado de autonomía y de apoyo mutuo que los países no podrán alcanzar en forma aislada. Por consiguiente, el orden internacional debe ofrecer un amplio espacio para el funcionamiento de instituciones regionales fuertes, evidentemente respetuosas de un orden global basado en reglas claras (es decir, un sistema de "regionalismo abierto", para utilizar un término proveniente de la literatura sobre integración económica). Efectivamente, las instituciones regionales pueden ser el mejor conducto para llevar a cabo el proceso de estructurar gradualmente un mejor orden internacional.

\section{III}

\section{Una visión amplia de la estabilidad macroeconómica y el papel de las políticas anticíclicas}

El concepto de estabilidad macroeconómica experimentó mutaciones importantes en el discurso económico en las dos últimas décadas. En el período de posguerra, dominado por el pensamiento keynesiano, este concepto se definía fundamentalmente en términos de pleno empleo y crecimiento económico estable, indudablemente acompañado de una baja inflación y cuentas externas sostenibles. Sin embargo, con el paso del tiempo el equilibro externo y la estabilidad de los precios pasaron al primer plano, mientras el énfasis keynesiano en la actividad real perdía importancia en el discurso e incluso tendía a desaparecer.

La consistencia que debe caracterizar a las políticas macroeconómicas debería apuntar a una definición amplia de estabilidad, en la que se tenga presente que no hay una correlación simple entre sus posibles definiciones y que pueden existir, por lo tanto, significativas disyuntivas de política. En esta materia, hay dos lecciones particularmente importantes. La primera es que la inestabilidad real tiene un costo muy elevado. Por este motivo, un concepto limitado de estabilización basada en un objetivo inflacionario puede tener un impacto tan negativo como las antiguas prácticas macroeconómicas en las que se subestimaba el costo de la inflación. Las recesiones provocan una gran pérdida de recursos, que puede tener efectos prolongados, entre otros una pérdida irreversible de activos empresariales, tanto tangibles como intangibles (conocimientos tecnológicos y organizacionales "tácitos", contactos comerciales, capital social acumulado y reputación 
de la empresa); una pérdida permanente del capital humano de los desempleados o subempleados, y una deserción escolar definitiva. La volatilidad del crecimiento genera una alta subutilización promedio de la capacidad productiva instalada, que reduce la productividad y las utilidades y, por ende, afecta adversamente la inversión (Ffrench-Davis, 1999). La incertidumbre que produce la inestabilidad de las tasas de crecimiento puede tener, así, efectos más severos sobre la acumulación de capital que una inflación moderada. De hecho, tal incertidumbre acentúa las estrategias microeconómicas "defensivas" (es decir, aquellas orientadas a proteger los activos de las empresas frente a un ambiente poco amigable) en vez de las estrategias "ofensivas", que conducen a altos niveles de inversión y de cambio tecnológico.

La segunda lección indica que los déficit del sector privado tienen un costo tan alto como los desequilibrios del sector público. Aun más, los balances de los agentes privados con un alto nivel de riesgo pueden ser tan nocivos como el desequilibrio de los flujos. En las economías liberalizadas desde el punto de vista financiero, los dos pueden interactuar en forma no lineal con choques que afecten la cuenta de capitales. La falta de una regulación y una supervisión prudenciales estrictas en los países, característica de las primeras etapas de la liberalización financiera, es un factor muy importante pero evidentemente no es el único. Los ciclos de auge y colapso son inherentes al funcionamiento de los mercados financieros. La expansión del gasto privado y los balances con un alto nivel de riesgo tienden a acumularse en los períodos de euforia financiera, dando origen a crisis cuando las condiciones excepcionales desaparecen. En la etapa de euforia se suele subestimar la posible inconsistencia intertemporal del gasto corriente y de las estructuras de financiamiento. Cuando las crisis involucran descalabros financieros tienen un costo extremadamente alto. La pérdida de activos puede implicar la pérdida de capital acumulado durante varios años. Para evitar una crisis sistémica, puede ser inevitable una socialización, bien sea parcial o significativa, de las pérdidas, pero ello afecta el desempeño fiscal (o cuasifiscal) futuro. La recuperación de la confianza en el sistema financiero es un proceso lento y el sector financiero mismo incrementa su aversión al riesgo, reduciendo su capacidad para desempeñar sus funciones económicas fundamentales.

Estas dos lecciones están estrechamente vinculadas, ya que los ciclos de auge y colapso financiero han sido la fuente predominante de los ciclos económicos en los países en desarrollo. Por consiguiente, la prin- cipal función de la política macroeconómica es el control de éstos con herramientas anticíclicas apropiadas. La experiencia de los países en desarrollo demuestra que el control de la volatilidad exige una combinación de tres conjuntos de políticas, cuya importancia relativa varía de acuerdo con las características estructurales y la tradición de política macroeconómica de cada país (Ocampo, 2000). El primero de ellos está integrado por políticas macroeconómicas - fiscales, monetarias y cambiarias - consistentes y flexibles, destinadas a evitar que los agentes públicos o privados acumulen deudas cuantiosas, y a que se produzcan desequilibrios en los precios macroeconómicos de mayor influencia (el tipo de cambio y las tasas de interés) y en el precio de los activos fijos y financieros. El segundo es un estricto sistema de regulación y supervisión prudenciales, con una clara orientación anticíclica. Esto significa que en los períodos de euforia financiera se deben aplicar normas más estrictas de regulación y supervisión financieras, para contrarrestar los riesgos cada vez mayores en que incurren los intermediarios financieros. El tercer elemento es una "política de pasivos" destinada a asegurar que se mantenga un perfil adecuado de vencimientos de las deudas interna y externa de los sectores público y privado. Las normas prudenciales sobre la cuenta de capital, es decir las aplicadas en períodos de euforia para evitar el endeudamiento excesivo, pueden jugar un papel esencial como política de pasivos, fomentando los flujos de largo plazo, y como instrumento que proporciona una mayor libertad para adoptar políticas monetarias anticíclicas.

El manejo de las políticas macroeconómicas anticíclicas no es una tarea fácil, ya que los mercados financieros generan fuertes incentivos a gastar en exceso durante los períodos de euforia financiera y, por el contrario, a ajustarse igualmente en exceso durante las crisis. Por otra parte, la globalización establece límites objetivos a la autonomía de los países y genera costos elevados a la pérdida de credibilidad cuando los instrumentos nacionales de política se manejan inadecuadamente. Por este motivo, puede ser necesario que el manejo de la política macroeconómica esté respaldado por instituciones e instrumentos de política que realcen la credibilidad, incluidos fondos de estabilización fiscal y bancos centrales independientes. En cambio, la renuncia explícita a la autonomía en materia de políticas (como la adopción de regímenes de convertibilidad o una moneda extranjera) dista mucho de ser una solución a este dilema. Por el contrario, predetermina la naturaleza de los ajustes, lo que, entre otras 
cosas, puede traducirse en ciclos económicos más intensos. En tal caso, es posible que el mercado no valide, mediante una reducción del riesgo país, el hipotético aumento de la "credibilidad" que se deriva de la renuncia a la autonomía en materia de política económica.

La solución más importante al dilema planteado por la falta de grados de libertad para adoptar una política macroeconómica anticíclica se encuentra, sin embargo, en el ámbito internacional (Eatwell y Taylor, 2000; Ocampo, 2001). Esto significa que una primera función esencial de las instituciones financieras internacionales, desde la perspectiva de los países en desarrollo, es precisamente compensar el efecto procíclico de los mercados financieros, suavizando en su origen los ciclos de auge y colapso financiero mediante una regulación adecuada, y ofreciendo mayores grados de libertad para que los países adopten políticas anticíclicas (mediante una vigilancia adecuada e incentivos que disminuyan los riesgos macroeconómicos y financieros en los períodos de euforia, y mecanismos que permitan suavizar los ajustes frente a interrupciones abruptas de los flujos de capital). Una segunda función, igualmente esencial, es ayudar a contrarrestar la concentración del crédito, poniendo recursos a disposición de los países y agentes que suelen tener un acceso limitado al crédito en los mercados privados de capital internacionales.

\section{IV}

\section{Las políticas macroeconómicas no son suficientes: el papel de las estrategias de desarrollo productivo}

La idea de que la combinación de una economía abierta y una macroeconomía estable —en el sentido limitado en que se utiliza actualmente este término, es decir como sinónimo de equilibrio fiscal y baja inflaciónpuede impulsar, por sí sola, el crecimiento económico, se ha visto frustrada hasta ahora. Esto ha llevado a un debate, aún no resuelto, sobre las causas de tal resultado. Según la interpretación ortodoxa, la liberalización de los mercados ha sido insuficiente. Esta interpretación se enfrenta, sin embargo, a la constatación de que la etapa de mayor crecimiento de los países en desarrollo en la posguerra y los episodios más prolongados de expansión acelerada (los "milagros" del sudeste asiático y, en los últimos años, de China y la India, así como los períodos de rápido crecimiento de Brasil y México en el pasado) no coinciden con las fases o episodios de mayor liberalización económica, incluso cuando se han basado en un amplio aprovechamiento de las oportunidades que ofrecían los mercados internacionales (lo que es un elemento más común, aunque tampoco generalizado).

Dos interpretaciones alternativas hacen hincapié en otros factores determinantes del crecimiento económico agregado o en fallas del mercado. En el primer caso, el lento crecimiento se atribuye a un desarrollo institucional o una acumulación de capital humano insuficientes. Estos factores son evidentemente esenciales, pero estas interpretaciones deben explicar por qué fue posible un crecimiento acelerado en períodos en los cuales ellos eran aún más limitados. En la segunda explicación se sostiene que deben adoptarse políticas "mesoeconómicas" de gran alcance para que los mercados liberalizados funcionen eficientemente, es decir políticas activas sobre competencia, regulación pública de mercados no competitivos o con grandes externalidades y corrección de las fallas en los mercados de factores, sobre todo en los de capital de largo plazo, tecnología, capacitación laboral y tierras. La adopción de políticas para corregir las fallas del mercado es una condición esencial para que éstos sean más eficientes y también puede influir favorablemente sobre la equidad, pero la relación entre dichas fallas y el crecimiento no es del todo clara. Desde este punto de vista, las fallas más importantes son posiblemente las que afectan a los mercados de capital de largo plazo y tecnología.

Las variantes históricas del pensamiento económico estructuralista, definido en términos amplios, ofrecen una alternativa más promisoria, que destaca la estrecha relación que existe entre la dinámica 
estructural, la inversión y el crecimiento económico. Acorde con esta corriente de pensamiento, el crecimiento económico no es un proceso lineal de expansión de "empresas representativas" o de incorporación de nuevas empresas de este tipo que producen un conjunto determinado de bienes en mayor escala, sino un proceso mucho más dinámico en el cual algunos sectores se expanden mientras otros se contraen, y algunas empresas avanzan mientras otras se estancan, transformando por completo las estructuras económicas. Este proceso involucra un fenómeno reiterado de "destrucción creativa", para usar la metáfora de Schumpeter (1962, cap. VIII). No todos los sectores tienen la misma capacidad de dinamizar la economía, de "propagar el progreso técnico", para emplear el concepto clásico desarrollado por Prebisch (1952). Debido a la complementariedad (externalidades) entre empresas y sectores productivos, los efectos macroeconómicos y distributivos pueden provocar una brusca aceleración del proceso de crecimiento o pueden detenerlo (Rosenstein-Rodan, 1943; Taylor, 1991; Ros, 2000), lo que da origen a sucesivas fases de desequilibrio, como lo señala el planteamiento clásico de Hirschman (1961). Dado que el conocimiento técnico y el conocimiento en general no se trasmiten por medio de manuales detallados, la trayectoria de crecimiento de las empresas pasa por un intenso proceso de adaptación y aprendizaje, intrínsecamente vinculado a la experiencia que se adquiere en el proceso productivo y que determina en gran medida la acumulación de conocimientos técnicos, comerciales y organizacionales (Katz, 1976; Amsden, 2001).

El postulado común de estas teorías es que el crecimiento económico está intrínsecamente vinculado al contexto estructural, constituido por el aparato productivo y tecnológico, la configuración de los mercados de productos y factores, las características de los agentes empresariales, y la forma en que estos mercados y agentes se relacionan con el contexto externo. En este caso, el liderazgo que ejercen algunos sectores y empresas es el factor dinámico esencial que da impulso al crecimiento económico. En los países en desarrollo, muchas de las fuerzas dinámicas están asociadas a la exitosa adaptación de actividades desarrolladas anteriormente en el mundo industrializado, ya sea a través de la sustitución de importaciones, la promoción de exportaciones o una combinación de ambas.

Aunque se podrían emplear formulaciones alternativas, una particularmente promisoria en términos de orientación de políticas se centra en dos conceptos esenciales: innovación y complementariedades (enca- denamientos). En este contexto, debe considerarse innovación toda actividad económica que aporta nuevas formas de hacer las cosas. La mejor definición de innovación fue proporcionada por Schumpeter (1961, cap. II) hace casi un siglo: nuevos bienes y servicios o nuevas características de éstos; nuevos métodos de producción o nuevas estrategias de comercialización; apertura de nuevos mercados; nuevas fuentes de materias primas, y nuevas estructuras de mercado. El segundo concepto destaca el papel de las sinergias estratégicas que determinan el grado de "competitividad sistémica" de las estructuras productivas pertinentes (CEPAL, 1990), a través de las externalidades que se crean entre los agentes económicos (Hirschman, 1961). La existencia de economías de escala dinámicas es la característica esencial de la innovación y su difusión, así como del desarrollo de complementariedades. El desarrollo institucional puede considerarse como una innovación, pero también como un componente esencial en el desarrollo de complementariedades.

Estas ideas han sido utilizadas recientemente por diferentes autores para resaltar la necesidad de una estrategia de desarrollo productivo como ingrediente fundamental de una economía en desarrollo dinámica y abierta, un tema antiguo de la literatura sobre "industrialización tardía" (o, mejor, de desarrollo tardío). Entre otros, Rodrik (1999) ha argumentado en favor de una "estrategia nacional de inversiones" que permita dar un impulso inicial al crecimiento, en tanto que la CEPAL se refiere a la necesidad de adoptar una estrategia de transformación estructural (CEPAL, 2000). Amsden (2001) destaca la función crucial de una estrecha colaboración entre el Estado y el sector empresarial, y la necesidad de "mecanismos de control recíproco" que establezcan un vínculo entre incentivos y resultados, para que la primera no se traduzca simplemente en una fuente de "captación de rentas".

Esta interpretación pone de relieve una de las características más importantes de los casos de desarrollo exitoso del pasado: un fuerte proceso de industrialización basado en una estrecha y firme colaboración entre el Estado y el sector privado. ¿Será posible lograr los mismos resultados con la apertura de los mercados mediante incentivos neutros, una relación débil entre el gobierno y el sector empresarial y la imposición de limitaciones multilaterales (Ronda Uruguay) a los instrumentos tradicionales de desarrollo? En otros términos, ¿será posible que la apertura de los mercados elimine la necesidad de adoptar políticas activas de desarrollo productivo? Queda por verse pero, en 
cualquier caso, los resultados alcanzados hasta el momento no han sido alentadores, aunque es posible que estén distorsionados por algunas de las características del proceso de transición. Los elementos "destructivos", derivados del cambio estructural adverso en la relación entre crecimiento y déficit comercial, y de la desintegración de los encadenamientos internos y los sistemas nacionales de innovación, han sido más fuertes que las oportunidades "creativas" que ofrece el acceso al mercado (aún insuficiente) y las innovaciones generadas por la expansión de las empresas multinacionales (Ocampo y Taylor, 1998; UNCTAD, 1999, cap. IV; CEPAL, 2000). En cualquier caso, si lo acontecido en el pasado es una buena guía y las interpretaciones estructurales son válidas, las estrategias explícitas de desarrollo destinadas a fomentar la innovación en el sentido amplio de la palabra y a crear complementariedades parecen ser una mejor alternativa, incluso para las economías abiertas de hoy. La comunidad internacional debería considerar dichas estrategias como un componente esencial de un desarrollo satisfactorio y seguir buscando instrumentos para aplicarlas, que no terminen dando origen a una competencia estéril entre los países con el fin de atraer industrias susceptibles de relocalización.

De parte de los países en desarrollo, se requiere un esfuerzo institucional y organizacional significativo para desarrollar los instrumentos adecuados para formular políticas activas de desarrollo productivo, ya que los viejos aparatos de intervención fueron desmantelados o debilitados significativamente durante la fase de liberalización en muchos (e incluso la mayoría) de los países. También se necesita un esfuerzo para diseñar instrumentos que, aparte de ser consistentes con las economías abiertas de hoy, eviten las "fallas de gobierno" que caracterizaron a algunas de ellas en el pasado —captación de rentas y "amiguismo" (cronyism).

La incorporación efectiva de la agenda de desarrollo sostenible es una demanda adicional sobre las estrategias de desarrollo productivo en la actualidad. De hecho, el alto grado de deterioro ambiental alcanzado por países en etapas intermedias o aun bajas de desarrollo indican que la sostenibilidad no puede ser vista de manera alguna como un lujo que puede posponerse. Este objetivo involucra mucho más que la conservación de la base de recursos naturales. Es, en esencia, un llamado a movilizar inversiones hacia sectores productivos dinámicos que utilicen tecnologías y procesos de producción limpia, en donde la competitividad se logre con la acumulación de capital en un sentido amplio - humano, social, físico y natural. La transición de posiciones reactivas a políticas activas en este frente por parte de los países en desarrollo es fundamental, como lo es su contrapartida: un mayor flujo de recursos desde las economías industrializadas para financiar la agenda ambiental global, de acuerdo con el principio de responsabilidades comunes pero diferenciadas.

\section{V}

\section{Mejorar los encadenamientos sociales}

En términos económicos, el progreso social puede concebirse como el producto de tres factores básicos: una política social de largo plazo, destinada a incrementar la equidad y garantizar la inclusión; un crecimiento económico que genere un volumen adecuado de empleos de calidad, y una reducción de la heterogeneidad estructural de los sectores productivos que reduzca las brechas productivas entre distintas actividades económicas y distintos agentes. Evidentemente, como se indica en la última sección de este ensayo, los criterios económicos no son los únicos que deben tenerse en cuenta en el diseño de la política social.

El Banco Mundial ha propuesto tres objetivos fundamentales para una estrategia de reducción de la pobreza: creación de oportunidades, seguridad y otorgamiento de espacios de poder (Banco Mundial, 2001). En una reformulación de estos objetivos, se podría argumentar que la equidad y la inclusión deben entenderse como un acceso amplio a los recursos, a las protecciones básicas, a la posibilidad de expresarse políticamente y a la participación. Un acceso equitativo a los recursos es la clave para una igualdad de oportunidades, no sólo en el terreno económico, sino también en el social, cultural y político. En el caso particular de la inversión en capital humano, resalta el carácter del gasto social como una inversión productiva. La protección es indispensable para evitar los "riesgos negativos" (enfermedad, vejez, desempleo y, el peor 
de todos, el hambre) e incentivar a la población y permitirle que asuma los "riesgos positivos", sobre todo los relacionados con el proceso de innovación. La protección contra los "riesgos negativos" es un elemento intrínseco del empleo de buena calidad. La capacidad de expresarse políticamente es esencial para que los intereses de los pobres sean debidamente tomados en cuenta en las decisiones que los afectan. La participación permite a los pobres convertirse en actores protagónicos en la creación de su propio futuro. En muchos contextos, las comunidades organizadas han demostrado ser un instrumento fundamental del cambio social y económico y, por lo tanto, un elemento central del desarrollo institucional.

Para alcanzar estos objetivos, la política social debe guiarse por tres principios básicos: universalidad, solidaridad y eficiencia (CEPAL, 2000, cap. 3). En los últimos años se ha producido una gran confusión con respecto a este tema, ya que los instrumentos - focalización, criterios de equivalencia entre contribuciones y beneficios, descentralización, participación del sector privado- y no los principios han guiado las reformas del sector social. Más aun, los principios rectores de las reformas hacen énfasis en el papel esencial de la política social como instrumento de cohesión (integración) social. Por lo tanto, los instrumentos deben estar claramente subordinados a los principios señalados. Así, la focalización debe considerarse un instrumento para lograr que los servicios básicos tengan una cobertura universal y nunca como un sustituto de la universalidad. Los criterios de equivalencia no deberían entrar en contradicción con el principio de solidaridad. Aplicados en forma adecuada, son un instrumento que contribuye a la eficiencia, al igual que la descentralización y la participación del sector privado.

Para alcanzar la equidad, la política social debe influir en los determinantes estructurales de la distribución del ingreso: educación, empleo, distribución de la riqueza y dependencia demográfica, así como sobre sus dimensiones étnicas y de género. Estos factores son la clave de la transmisión intergeneracional de la desigualdad y la pobreza. Por lo tanto, romper estos encadenamientos intergeneracionales es la clave de una estrategia social exitosa. Esto debe reflejarse particularmente en políticas integradas de apoyo a los más pobres.

La educación es la clave de un crecimiento equitativo, especialmente en la era de la información y el conocimiento, pero sus objetivos van más allá de sus dimensiones de "capital humano": es también un elemento decisivo del desarrollo democrático y de una ciudadanía sólida y, en términos más amplios, de la realización personal. Sus efectos sobre la equidad han sido sobredimensionados en los debates recientes sobre el tema. En una sociedad altamente segmentada, la educación también es un instrumento de segmentación. Esto debe tenerse en cuenta si se usa para mejorar los niveles de equidad. Más aun, una generación inadecuada de empleos de buena calidad puede derrotar los esfuerzos que se realicen en el ámbito de la educación, tanto en términos de acumulación de capital humano (que en situaciones extremas emigra y en circunstancias más normales queda subempleado) y de equidad (la segmentación laboral multiplica los efectos de la segmentación educacional). El nexo entre crecimiento económico y progreso social es, por lo tanto, particularmente crítico en este contexto. De hecho, éste, al igual que otros encadenamientos que mencionaremos más adelante, resaltan claramente el hecho de que la política social no es suficiente por sí misma: para que rinda sus frutos debe estar apoyada tanto por una buena macroeconomía como por estrategias activas de desarrollo productivo.

En el entorno de cambio acelerado que caracteriza a las economías modernas, la capacidad de adaptación de la mano de obra al cambio tecnológico y al ciclo económico adquiere cada vez más importancia. Los factores que contribuyen en forma más decisiva a dicha adaptabilidad son los buenos programas de capacitación de mano de obra; las instituciones que favorecen la cooperación, tanto a nivel nacional (diálogo social) como en las empresas; una adecuada protección social, tanto de carácter permanente como aquella dirigida a hacer frente a circunstancias adversas, y una política prudente de salario mínimo. La flexibilidad puede contribuir también a este resultado, si está acompañada de una mayor protección, pero es apenas uno de los instrumentos alternativos. Al respecto, es necesario tener en cuenta que los mercados laborales más flexibles pueden influir negativamente sobre otros factores que tienen efectos favorables sobre la adaptabilidad laboral, particularmente sobre la cooperación entre los trabajadores y las empresas. Aun más importante, la flexibilidad no debe considerarse un sustituto de políticas macroeconómicas inadecuadas. De hecho, en un contexto macroeconómico inestable o en condiciones de crecimiento económico lento, la generación de empleo será inevitablemente limitada y una mayor flexibilidad puede traducirse en un rápido deterioro de la calidad del mismo. En otras palabras, la flexibilidad tiene externalidades negativas (afecta puestos de trabajo que de otra manera serían estables) que no deben ignorarse. 
El crecimiento económico lento afecta a la equidad por otro canal de importancia decisiva en los países en desarrollo: incrementa la heterogeneidad estructural. Esta expresión, utilizada por la escuela estructuralista latinoamericana, es preferible a la de "dualismo", porque la heterogeneidad que caracteriza a los países y las sociedades en desarrollo no puede describirse como una dualidad entre un "sector moderno" y un "sector tradicional" y porque los sectores de baja productividad se crean y transforman constantemente y sólo unos pocos segmentos que van quedando rezagados pueden definirse como "tradicionales". Esto fue lo que aconteció en América Latina en los años noventa: la región generó más empresas de "clase mundial", capaces de integrarse exitosamente a la economía mundial, muchas de ellas filiales de empresas trasnacionales; al mismo tiempo aumentaron las actividades de baja productividad, que absorbieron a siete de cada diez trabajadores durante la etapa de expansión que culminó con la crisis asiática (CEPAL, 2000, cap. 1). En realidad, no existen mecanismos automáticos que garanticen que la rápida innovación tecnológica en sectores dinámicos fomente un crecimiento económico acelerado. En ausencia de encadenamientos internos adecuados o cuando predominan los efectos "destructivos" de la reestructuración productiva y las estrategias microeconómicas defensivas que los acompañan, puede simplemente acentuar la heterogeneidad estructural. Si esto acontece, los efectos sobre el crecimiento económico serán débiles y se experimentarán presiones adicionales sobre la equidad.

Los encadenamientos entre la modernización de los sectores líderes y el resto de la economía son, por lo tanto, importantes, no sólo para el crecimiento sino también para la equidad. Las estrategias de desarrollo productivo pueden jugar un papel trascendental en ambas dimensiones. Esto resalta igualmente el papel de una buena distribución de los activos productivos. De hecho, la evidencia demuestra que una buena distribución de activos, que genera un universo de empresas pequeñas sólidas, está asociada con una mejor distribución del ingreso y una menor concentración del poder en general. Por lo tanto, las políticas destinadas a democratizar el acceso a los activos productivos - capital, tecnología, capacitación y tierras- juegan un papel trascendental, tanto en términos de crecimiento como de equidad. Entre estas políticas se cuentan las de desarrollo rural y aquellas destinadas a formalizar las microempresas. Estas políticas deben complementarse con una expansión gradual de los sistemas de seguridad social a los trabajadores de pequeñas empresas y a quienes laboran por cuenta propia.

La interacción entre el capital humano y el empleo de buena calidad y los efectos de una mejor distribución de los activos productivos son sólo algunos de los nexos positivos entre desarrollo y equidad. También puede haber encadenamientos favorables de economía política, efectos positivos en el mercado de capitales y nexos entre cohesión social, inversión y productividad. Las relaciones entre equidad y desarrollo fueron uno de los temas favoritos de la literatura sobre desarrollo de los años sesenta. Afortunadamente, han vuelto a ocupar un primer plano en el pensamiento económico (véase en Aghion, Caroli y García-Peñalosa, 1999, una reseña reciente sobre la materia).

Dada la innegable relación existente entre desarrollo económico y desarrollo social, es indispensable diseñar marcos integrados de política. Estos marcos deben considerar explícitamente las relaciones mencionadas, al igual que aquellas que existen al interior de las políticas sociales (refuerzo mutuo entre distintas políticas sociales, sobre todo a través de programas integrados de erradicación de la pobreza) y entre las políticas económicas (conexiones entre la macro y la mesoeconomía, especialmente para facilitar el desarrollo de sectores dinámicos de pequeñas empresas). Uno de los puntos más débiles en esta esfera es la falta de instituciones que permitan la adopción de marcos integrados de política. Las instituciones correspondientes deberían caracterizarse por una activa participación de distintos actores sociales, con una capacidad efectiva de intervención de los sectores pobres; sistemas eficaces de coordinación entre las autoridades económicas y sociales, en los que las prioridades sociales se incorporen efectivamente a la política económica; y normas que den "visibilidad" a los efectos sociales de las políticas económicas. Esto último exige, entre otras cosas, el examen periódico de dichos efectos por las autoridades macroeconómicas, incluidos los bancos centrales; normas que dispongan que los proyectos de ley de presupuesto incorporen un análisis de los efectos distributivos del gasto propuesto, los cuales deben tomarse en cuenta para su aprobación en el Congreso; prácticas similares en relación con los proyectos de reforma tributaria, etc. 


\section{VI}

\section{Objetivos más amplios}

Uno de los cambios más positivos de la última década fue el pleno reconocimiento de que el desarrollo tiene objetivos más amplios (Stiglitz, 1998). El concepto de "desarrollo humano" y el concepto más reciente de "desarrollo como libertad" (Sen, 1999) son expresiones de esta perspectiva, pero ella tiene indudablemente raíces profundas en los debates sobre desarrollo. Su principal manifestación es la difusión gradual de ideas y valores globales, entre ellos los de derechos humanos, desarrollo social, equidad de género, respeto de la diversidad étnica y cultural, y protección del medio ambiente. La mejor expresión de esta "globalización de los valores" es la serie de declaraciones de las cumbres de las Naciones Unidas realizadas en los años noventa, incluida la Declaración del Milenio. Los valores globales, y sobre todo, los derechos humanos, en su doble dimensión de derechos civiles y políticos, por una parte, y de derechos económicos, sociales y culturales, por otra, deberían considerarse hoy como el marco ético para la formulación de las políticas de desarrollo.

Las consecuencias de esta nueva perspectiva son más profundas de lo que la mayoría de los economistas están dispuestos a aceptar. Siguiendo a Polanyi (1957), significa fundamentalmente que el sistema económico debe estar subordinado a objetivos sociales más amplios. Un tema que ha venido adquiriendo cada vez más importancia en este sentido es la necesidad de enfrentar las poderosas fuerzas centrífugas que caracterizan actualmente al ámbito privado. Efectivamente, en muchos países en desarrollo (e industrializados), la población viene perdiendo el sentido de pertenencia a la sociedad y de identificación con pro- pósitos colectivos y la necesidad de crear lazos de solidaridad. Este hecho pone de manifiesto la importancia de fomentar esos lazos, con el fin de "crear sociedad", una conciencia más amplia de las responsabilidades sociales de los individuos y los grupos. La iniciativa correspondiente puede provenir del Estado o de la sociedad civil. En este sentido, como lo señalamos al comienzo de este ensayo, el ámbito de "lo público" debe concebirse como el punto de encuentro de los intereses colectivos más que como un sinónimo de las actividades del Estado. En otras palabras, todos los sectores de la sociedad tienen que participar más activamente en las instituciones políticas democráticas, desarrollando una amplia gama de mecanismos, dentro de la sociedad civil, para fortalecer las relaciones de solidaridad y responsabilidad social y, sobre todo, consolidar una cultura de desarrollo colectivo basada en la tolerancia frente a la diferencia y en la disposición al compromiso.

Los enormes desafíos intelectuales y las tareas prácticas que supone el reconocimiento de estos factores nos lleva a concluir con una nota de humildad. Creer que "ya sabemos lo que se debe hacer" es simplemente una expresión de arrogancia de los economistas, que incluso se ha acentuado con el predominio de las visiones ortodoxas del pensamiento sobre el desarrollo desde los años ochenta. Las reflexiones sobre los resultados frustrantes de las reformas y el descontento social deberían convencer a muchos de la necesidad de repensar la agenda de desarrollo. Y así está sucediendo, pero éste es apenas un proceso en marcha, aún incompleto.

Bibliografía

Aghion, Ph., E. Caroli y C. García-Peñalosa (1999): Inequality and economic growth: the perspective of the new growth theories, Journal of Economic Literature, vol. 37, $\mathrm{N}^{\circ} 1$, Nashville, Tennessee, American Economic Association, diciembre.

Amsden, A. (2001): The Rise of "The Rest" : Challenges to the West from Late Industrializing Countries, Nueva York, Oxford University Press.

Banco Mundial (2001): Informe sobre el desarrollo mundial 2000/ 2001: Lucha contra la pobreza, Washington, D.C.

CEPAL (Comisión Económica para América Latina y el Caribe) (1990): Transformación productiva con equidad. La tarea prioritaria del desarrollo de América Latina y el Caribe en los años noventa, LC/G.1601-P, Santiago de Chile, marzo. (2000): Equidad, desarrollo y ciudadanía (LC/G.2071/Rev.1-P), Santiago de Chile.

Cornia, G. A. (1999): Liberalization, globalization and income distribution, Working paper, $\mathrm{N}^{\circ} 157$, Helsinki, Universidad de las Naciones Unidas (UNU)/Instituto Mundial de Investigaciones de Economía del Desarrollo (WIDER), marzo.

Eatwell, J. y L. Taylor (2000): Global Finance at Risk: The Case for International Regulation, Nueva York, The New Press.

Ffrench-Davis, R. (1999): Macroeconomía, comercio y finanzas para 
reformar las reformas en América Latina, Santiago de Chile, McGraw-Hill Interamericana.

Helleiner, G.K. (2000): Markets, politics and globalization: can the global economy be civilized?, The Tenth Raúl Prebisch Lecture, Ginebra, UNCTAD, 11 de diciembre.

Hirschman, A.O. (1961): La estrategia del desarrollo económico, México, DF, Fondo de Cultura Económica.

Katz, J. (1976): Importación de tecnología, aprendizaje e industrialización dependiente, México, Fondo de Cultura Económica.

Kaul, I., I. Grunberg y M.A. Stern, comps. (1999): Global Public Goods. International Cooperation in the $21^{\text {st }}$ Century, Programa de las Naciones Unidas para el Desarrollo (PNUD), Nueva York, Oxford University Press.

Naciones Unidas (2000): Declaración del Milenio, Cumbre del Milenio 2000, Nueva York, 6 a 8 de septiembre.

Ocampo, J.A. (2000): Developing Countries' Anti-Cyclical Policies in a Globalized World, Temas de Coyuntura, $\mathrm{N}^{\circ} 13$, Santiago, Chile, CEPAL, octubre.

(2001): Recasting the international financial agenda, en J. Eatwell y L.Taylor (comps.), External Liberalization, Economic Performance, and Social Policy, Nueva York, Oxford University Press, en prensa.

Ocampo, J.A. y L. Taylor (1998): Trade liberalisation in developing economies: modest benefits but problems with productivity growth, macro prices, and income distribution, The Economic Journal, vol. 108, $\mathrm{N}^{\circ} 450$, Oxford, Reino Unido, Basil Blackwell, septiembre.

PNUD (Programa de las Naciones Unidas para el Desarrollo) (1999): Informe sobre desarrollo humano, 1999, Nueva York.

Prebisch, R. (1952): Problemas teóricos y prácticos del crecimiento económico, E/CN.12/221, México, D.F., Comisión Económica para América Latina (CEPAL).
Polanyi, K. (1957): The Great Transformation: the Political and Economic Origins of Our Time, Boston, Beacon Press.

Rodrik, D. (1997): Has Globalization Gone Too Far?, Washington, D.C., Institute for International Economics (IIE).

(1999): The New Global Economy and the Developing Countries: Making Openness Work, Policy Essay, $\mathrm{N}^{\circ} 24$, Washington, D.C., Consejo de Desarrollo de Ultramar.

Ros, J. (2000): Development Theory and The Economics of Growth, Ann Arbor, The University of Michigan Press, septiembre.

Rosenstein-Rodan, P.N. (1943): Problems of industrialization of Eastern and South-Eastern Europe, The Economic Journal, vol. 53, Oxford, Reino Unido, Basil Blackwell, junio-septiembre.

Schumpeter, J. (1961): The Theory of Economic Development, Oxford, Oxford University Press.

(1962): Capitalism, Socialism and Democracy, tercera edición, Nueva York, Harper Torchbooks.

Sen, A. (1999): Development as Freedom, Nueva York, Alfred A. Knopf.

Stiglitz, J. A. (1998): "More instruments and broader goals: moving toward the post-Washington Consensus", WIDER Annual Lectures 2, Helsinki, enero.

Sunkel, O. (1991): El desarrollo desde dentro: un enfoque neoestructuralista para la América Latina, Lecturas, $\mathrm{N}^{\circ}$ 71, México, D.F., Fondo de Cultura Económica.

Taylor, L. (1991): Income Distribution, Inflation and Growth, Cambridge, Massachusetts, The MIT Press.

UNCTAD (Conferencia de las Naciones Unidas sobre Comercio y Desarrollo) (1999): Informe sobre el comercio y el desarrollo, 1999, Ginebra.

Williamson, J. (1998): The Washington Consensus revisited, en Louis Emmerij (comp.), Economic and Social Development into the XXI Century, Washington, D.C., Banco Interamericano de Desarrollo (BID). 